\title{
Creative Individuals are Social Risk-takers: Relationships between Creativity, Social Risk-Taking and Fear of Negative Evaluations
}

Eric Bonetto

Aix Marseille Univ, LPS, Aix-en-Provence, France

Aix Marseille Univ, PSYCLE (Center for Research on the Psychology of Cognition, Language and Emotion), Aix-en-Provence, France InCIAM (Institute of Creativity and Innovation of Aix-Marseille), Aix-en-Provence, France

E-mail address: bonetto.ericbw@gmail.com

\section{Nicolas Pichot}

Aix Marseille Univ, PSYCLE (Center for Research on the Psychology of Cognition, Language and Emotion), Aix-en-Provence, France InCIAM (Institute of Creativity and Innovation of Aix-Marseille), Aix-en-Provence, France

E-mail address: nicolas.pichot@univ-amu.fr

\section{Jean-Baptiste Pavani}

Aix Marseille Univ, PSYCLE (Center for Research on the Psychology of Cognition, Language and Emotion), Aix-en-Provence, France

E-mail address: jean-baptiste.PAVANI@univ-amu.fr

\section{Jaïs Adam-Troïan}

American University of Sharjah, United Arab Emirates

E-mail address: jadam @ aus.edu

\section{ARTICLE INFO}

\section{Keywords:}

Creativity

Creative self-beliefs

Creative achievement

Social risk-taking

Fear of negative evaluation

\section{A B S TRACT}

If the importance of social psychological factors in creativity has been widely emphasized, research across the social sciences mainly focused on identifying contextual factors that increase or decrease individuals' creative behaviors. Few studies have investigated the relationships between creativity and individual characteristics related to interpersonal and social interactions. The present contribution aimed to consider such characteristics, namely social risktaking (i.e., willingness to challenge norms) and fear of negative evaluation (i.e., apprehension about receiving negative judgments of others). Two correlational studies were therefore implemented in order to clarify previous research suggesting that creativity would be positively associated with social risk-taking and to extend them by testing potential associations between creativity and fear of negative evaluation. Using self-ratings of creativity, but also creative self-beliefs and creative achievement, we hypothesized that creativity would correlate positively with social risk likelihood and negatively with fear of negative evalua- 


\section{Article history:}

Received 17 August 2020

Received in revised form 11 November 2020

Accepted 24 November 2020

ISSN: 2354-0036

DOI: $10.2478 /$ ctra-2020-0016 tion. As predicted, and replicating previous results, all our measures of creativity correlated positively with social risktaking. Our results also consistently highlighted a negative association between beliefs in one's creative capacities (creative self-efficacy) and one's level of fear of negative evaluation. These findings corroborate the idea according to which creative individuals would be (social) risk-takers. In addition, individuals who are confident in their ability to generate creative productions would be less afraid of negative judgments from others. Several perspectives are proposed, in particular in terms of the intervention designed in order to enhance individuals' creativity.

\section{INTRODUCTION}

Creativity, which is production of novel and useful ideas (Runco \& Jaeger, 2012), involves specific combinations of knowledge, abilities, and intellectual skills (Watts, Steel, \& Song, 2017). Consequently, investigating associations between creativity and intellectual abilities and personality traits has been - and still is - the focus of numerous studies. Creativity has been found to be positively correlated with intelligence itself (Furnham \& Bachtiar, 2008; Karwowski et al., 2016; Kim, 2005) or with such correlates of intellectual ability, as need for cognition (e.g., Dollinger, 2003). Personality traits have also been found to be associated with creativity (Furnham \& Bachtiar, 2008; Kaufman et al., 2016). Especially, openness to experience was found to be the strongest predictor of creativity (followed by extraversion, conscientiousness, neuroticism, and agreeableness; see e.g., Karwowski \& Lebuda, 2016).

Researchers have also widely emphasized the importance of social psychological factors in creativity (e.g., Amabile, 1996, 2018; Amabile \& Pillemer, 2012; Csikszentmihalyi, 2015), principally through consideration of contextual factors influencing creative behaviors. In particular, half a century of research across the social sciences focused on identifying contextual factors that increase or decrease individuals' creative behaviors (Lucas \& Nordgren, 2020; see Amabile, 1996, 2018; Haslam, Adarves-Yorno, Postmes, \& Jans, 2013; Mumford, 2012; Sternberg, 1999).

However, as a "compromise" between these two focuses (the study of individual traits and that of social factors), few studies investigated the relationships between creativity and individual characteristics related to interpersonal and social interactions. Indeed, creative individuals often present their ideas or productions to others for approval, adoption, appreciation, or criticism. Presenting such ideas or productions to others is socially risky, since there is always a chance that one's creative - hence counter normative - solution might trigger negative peer evaluations (Tyagi, Hanoch, Hall, Runco, \& Denham, 2017). Thus, previous results suggest that creativity is associated with risk-taking tendencies in the social domain. More precisely, Tyagi et al. (2017) showed that self-rating of 
creativity (i.e., self-report measure of creativity), creative ideation (i.e., measure of ideation ability; Runco, Plucker, \& Lim, 2001), and creative personality (Gough, 1979) were all positively associated with social risk-taking (i.e., willingness to challenge norms). However, their results indicated no association between such risk-taking and measures of divergent thinking (Guilford, 1967), which contradicted established associations between social risk-taking and creative achievement (Carson, Peterson, \& Higgins, 2005). These contrasting results plead in favor of further investigating the link between creativity and social risk-taking.

In fact, it is worth noting that risk-taking involves potential negative consequences or losses in the social domain (Ben-Zur \& Zeidner, 2009). These consequences may refer to negative evaluations from others leading, for instance, to social exclusion, sometimes at the cost of one's life (Bonetto, Pichot, Pavani, \& Adam-Troïan, 2021). Given these potentially huge costs, it is possible that creative individuals may display lower apprehension about receiving negative judgments or being rejected. This hypothesis is in line with the idea according to which social sanctions (including negative judgments) could inhibit individuals' creative behaviors, so that lower apprehension of sanctions may be a necessary enabler of creative production (Bonetto et al., 2021). Thus, studying the role of social risktaking in creativity requires consideration of individuals' sensitivity - or apprehension - of negative evaluation from others. Yet, such fear of negative evaluation (Leary, 1983) has received very little attention in the creativity literature so far.

Accordingly, these considerations point at the importance of considering both social risk-taking and fear of negative evaluation in the study of creativity. Two correlational studies were therefore implemented in order to (1) clarify Tyagi et al.'s (2017) findings, and to (2) extend them by testing potential associations between creativity and fear of negative evaluation. Effect sizes were interpreted in light of Funder and Ozer's (2019) guidelines for psychological research. Data and materials are openly available here: https://osf.io/hdxeu/?view_only=17396261cee442439d7ca90074527c35. The studies were conducted in accordance with the 1964 Helsinki declaration (WMO, 1964) and its later amendments, the ethical principles of the French Code of Ethics for Psychologists (CNCDP, 2012) and the 2016 APA Ethical Principles of Psychologists and Code of Conduct (APA, 2017).

\section{STUDY 1}

\section{Method and Hypotheses}

This first study aimed to replicate the association between social risk-taking and creativity measured through a self-rating measure (as in Tyagi et al., 2017), and to confirm this re- 
sult through the measure of creative self-beliefs (individuals' beliefs about their creative abilities; Karwowski \& Lebuda, 2016). We hypothesized that all our measures of creativity would correlate positively with social risk likelihood $(\mathrm{H} 1)$. In addition, this study aimed to extend Tyagi et al's results by testing the association between creativity and individual's apprehension of being negatively evaluated. We hypothesized that all our measures of creativity would correlate negatively with fear of negative evaluation $(\mathrm{H} 2)$.

\section{Participants}

An online questionnaire was distributed among randomly selected social network groups (e.g., trade, sales advertisements, hobby, and community pages). The final sample consists of 258 French people $(8.90 \%$ male, $1.60 \%$ other; Mage $=27.53, S D=11.70)$.

\section{Measurements}

Self-Rating of creativity (SR; adapted from Furnham \& Bachtiar, 2008) was a single-item self-rating measure of creativity (from 1 "Not at all creative" to 7 "totally creative"). We also used the Short Scale for Creative Self (Karwowski, 2012), which includes a 5-item measure of Creative Personal Identity (CPI; the importance of creativity for self-definition) and a 6-item measure of Creative Self-efficacy (CSE; the belief in one's creative capacities). All items were rated on a 7-point Likert scale (from 1 "Definitely not" to 7 "Definitely yes"). The structure of the scale was examined by a confirmatory factor analysis. The two -factor solution $\left(x^{2}(43)=259.50, p<.001\right.$; CFI = .89; TLI = .86; SRMR = .08; RMSEA $=.14,90 \% \mathrm{Cl}[.12, .16])$ fitted the data better than the one-factor one $\left(X^{2}(44)=298.00\right.$, $p<.001 ; \mathrm{CFI}=.87 ; \mathrm{TLI}=.83 ; \mathrm{SRMR}=.08 ; \mathrm{RMSEA}=.15,90 \% \mathrm{Cl}[.13, .17])$. Internal consistency of both scales was high $\left(\alpha_{\text {Creative Personal Identity }}=.91, \alpha_{\text {Creative Self-Efficacy }}=.81\right)$. CPI and CSE displayed a very strong positive correlation $\left(r=.72, \mathrm{BF}_{10}>100\right)$.

Social risk likelihood was assessed through the dedicated 6-item scale extracted from the Domain Specific Risk-taking Scale (Blais \& Weber, 2006). Participants reported their answers using a 7-point Likert scale (from 1 "Extremely unlikely" to 7 "Extremely likely"). The scale displayed adequate internal consistency $(\alpha=.66$, one item dropped due to poor item-rest correlation; see supplementary materials).

The Brief Fear of Negative Evaluation II (Carleton, Collimore, \& Asmundson, 2007) is an 8-item measure of individual's tolerance of the possibility that they may be judged in a disagreeable or hostile way by others. Participants reported their answers using a 7-point Likert scale (from 1 "Definitely not" to 7 "Definitely yes"; $\alpha=.95$ ).

\section{Results}

Descriptive statistics and Bayesian correlations are presented in Table 1. We observed strong intercorrelation among all our measures of creativity (from $r=.72$ to $r=.81$, all 
p's < .001). We also found a medium-size negative association between fear of negative evaluation and social risk likelihood $(r=-.26, p<.001)$. In addition, in line with Tyagi et al.'s (2017) findings, our measures of creativity displayed medium-to-strong associations with social risk likelihood (from $r=.26$ to $r=.38$, all $p$ 's $<.001$ ), providing support for $\mathrm{H} 1$. We also found a medium negative correlation between CSE and fear of negative evaluation ( $r=-$ $.20, p<.01)$. However, no associations between fear of negative evaluation and other measures of creativity (i.e., SR and CPI) were observed. $\mathrm{H} 2$ is thus partially corroborated.

Table 1

Mean, standard deviation, and correlation of all variables $(N=258)$

\begin{tabular}{lrrrrrr}
\hline & $M(S D)$ & 1 & 2 & 3 & 4 & 5 \\
\hline 1. SR & $4.24(1.56)$ & - & & & & \\
2. CPI & $4.82(1.47)$ & $.81^{* * *}$ & - & & & \\
3. CSE & $4.62(1.07)$ & $.73^{* * *}$ & $.72^{* * *}$ & - & & \\
4. Risk likelihood & $5.29(0.98)$ & $.27^{* * *}$ & $.26^{* * *}$ & $.38^{* * *}$ & - & \\
5. Fear neg. eval. & $4.43(1.67)$ & -.12 & -.03 & $-.20^{* *}$ & $-.26^{* * *}$ & - \\
\hline
\end{tabular}

Note. SR: Self-Rating of creativity; CPI: Creative Personal Identity; CSE: Creative Self-efficacy; Fear neg. eval.: Fear of negative evaluation; ${ }^{\star *} p<.01,{ }^{* \star *} p<.001$

\section{Discussion}

Results from this study tend to confirm those obtained by Tyagi et al. (2017). As predicted, all our measures of creativity correlated positively with social risk likelihood $(\mathrm{H} 1)$. Moreover, results also partially support H2. Indeed, it seems that strong beliefs in one's creative capacities were associated with weaker apprehension of negative evaluations. However, this result has been observed with a general measure of fear of negative evaluation, not with a measure adapted to the context of creativity. Moreover, given the contrasting results observed by Tyagi et al. (2017) about the association between social risktaking and creative achievement, it would be appropriate to further examine this association here. A second study was designed in order to further corroborate these findings through consideration of these two points.

\section{STUDY 2}

\section{Method and Hypotheses}

A second correlational study was thus implemented and it included measures of fear of negative evaluation of new ideas/productions (individuals' general tendency to apprehend negative evaluations about their idea/production) and of negative evaluation of the individual (individuals' general tendency to apprehend negative evaluations about themselves). We hypothesized that all our measures of creativity would correlate positively 
with social risk likelihood. We also hypothesized that they would correlate negatively with both measures of fear of negative evaluation.

\section{Participants}

An online questionnaire was distributed among social network groups (e.g., trade and sales advertisements groups). The global sample consists of 327 French people (11\% male, $.61 \%$ other; Mage $=41.26, S D=14.42)$.

\section{Measurements}

SR, CSE $(\alpha=.86)$ and social risk likelihood $(\alpha=.65$, one item dropped due to poor itemrest correlation; see supplementary materials) were assessed through the same scales as in Study 1. Since no correlation was found between CPI and fear of negative evaluation, CPI was not included in this study. The Biographical Inventory of Creative Behaviors (BICB; Batey \& Furnham, 2008; Furnham \& Bachtiar, 2008) assessed everyday creativity and creative achievement. Participants indicated, from a list of 34 activities, those in which they had been actively involved over the past 12 months $(\alpha=.75)$.

Participants were also asked to indicate the extent to which, when they introduce a new idea or production to others, they generally tend to apprehend negative evaluations about themselves (as individuals) versus about this idea/production. Fear of negative evaluation of new ideas/productions was assessed through three items adapted from the scale used in the previous study $(\alpha=.86)$. Fear of negative evaluation of the individual was assessed through the same three items adapted to the evaluation of the individual $(\alpha=.89)$. The items were rated on a 7-point Likert scale (from 1 Definitely not to 7 Definitely yes).

\section{Results}

Descriptive statistics and Bayesian Pearson correlations are presented in Table 2. We observed strong intercorrelation between all our measures of creativity (from $r=.40$ to $r=.80$, all $p$ 's <.001). We also found a medium-size negative association between social risk likelihood and fear of negative evaluation of both the individual $(r=-.19, p<.001)$ and new ideas $(r=-.17, p<.01)$. In line with Study 1's results, our measures of creativity displayed medium-to-strong associations with social risk likelihood (from $r=.15$ to $r=.33$, all p's <.01), providing support for $\mathrm{H} 1$.

We observed negative correlations between CSE and fear of negative evaluation of the new ideas $(r=-.18, p<.01)$ and the individual $(r=-.21, p<.001)$. We also observed a negative association between SR and fear of negative evaluation of the individual $(r=-.14, p<.05)$, but not new ideas. However, no evidence of associations between BICB scores and both measures of fear of negative evaluation was observed. $\mathrm{H} 2$ is thus partially corroborated. 
Table 2

Mean, standard deviation, and correlation of all variables $(N=327)$

\begin{tabular}{|c|c|c|c|c|c|c|c|}
\hline & $M(S D)$ & 1 & 2 & 3 & 4 & 5 & 6 \\
\hline 1. $S R$ & $4.48(1.75)$ & - & & & & & \\
\hline 2. CSE & $4.91(1.24)$ & $.80 * * *$ & - & & & & \\
\hline 3. $\mathrm{BICB}$ & $6.04(3.78)$ & $.40 * * *$ & $.44 * * *$ & - & & & \\
\hline 4. Risk Likelihood & $5.37(1.09)$ & $.29 * * *$ & $.33 * * *$ & $.15^{* *}$ & - & & \\
\hline 5. Fear neg. eval. new ideas & $3.90(1.77)$ & -.11 & $-.18 * *$ & -.07 & $-.17 * *$ & - & \\
\hline 6. Fear neg. eval. individual & $3.85(1.84)$ & $-.14 *$ & $-.21 * * *$ & -.09 & $-.19 * * *$ & $.82 * * *$ & - \\
\hline
\end{tabular}

Note. SR: Self-Rating of creativity; CSE: Creative Self-efficacy; Fear neg. eval. new ideas: Fear of negative evaluation of new ideas/productions; Fear neg. eval. individual: Fear of negative evaluation of the individual; ${ }^{*} p<.05,{ }^{* *} p<.01,{ }^{* * *} p<.001$

\section{Discussion}

These results mainly confirm Study 1's findings related to the positive association between creativity and social risk-taking. More precisely, all our measures of creativity (SR, self-beliefs, achievement) correlated positively with social risk-taking $(\mathrm{H} 1)$. They also allow to be more confident about the presence of a positive correlation between social risktaking and creative achievement, despite a smaller effect size observed here. Moreover, once again, it seems that strong belief in one's creative capacities was associated with weaker apprehension of negative evaluations $(\mathrm{H} 2)$.

\section{General Discussion}

Across our studies, and as expected $(\mathrm{H} 1)$, we observed that all our measures of creativity correlated positively with social risk-taking. These results replicated Tyagi et al.'s (2017) findings, and seem to corroborate the idea that creative individuals display stronger levels of social risk-taking (i.e., willingness to challenge norms). In other words, these findings corroborate the idea, according to which creative individuals would be (social) risk-takers.

Importantly, and as predicted, our results consistently highlighted a negative association between beliefs in one's creative capacities and one's level of apprehension at the prospect of being negatively evaluated (H2). Thus, strong beliefs in one's creative capacities seem to be associated with weaker apprehension of negative evaluation. No such association had been previously observed between this fear of negative evaluation and other measures of creativity. Accordingly, individuals who are confident in their ability to generate creative productions seem to be less afraid of negative judgments from others. More precisely, such individuals would judge themselves to be more capable of creating productions that are valuable to the community (Runco \& Jaeger, 2012), which 
seems to be associated with considering these productions and themselves as less likely to be the object of negative reactions and to rejection. This finding is thus in line with the idea according to which creative individuals may display lower apprehension about receiving social sanctions, here negative evaluations from others (Bonetto et al., 2021). It is worth noting that, while positive association between creative achievement and social risk -taking is observed (supporting $\mathrm{H} 1$ ), no correlation is observed between these achievements and the fear of evaluation (contrary to $\mathrm{H} 2$ ).

Furthermore, it is worth noting that all our measures of creativity on the one hand, but also our measures of social risk-taking and fear of negative evaluation on the other hand, intercorrelated in a coherent way, which makes us confident in the validity of our results (for correlations between creativity measures, see Furnham \& Bachtiar, 2008; Karwowski \& Barbot, 2016). In addition, the effects observed here present sizes in line with effect sizes previously observed for correlations between creativity and other individual characteristics, such as intellectual abilities or personality traits (e.g., Dollinger, 2003; Karwowski et al., 2016; Karwowski \& Lebuda, 2016; Kim, 2005).

The results presented here provide some perspectives. They first argue for a more refined study of the association between creative achievements and fear of negative evaluation. Rather than a relationship between creativity and fear of negative evaluation, it could be hypothesized that this same apprehension is associated with the propensity to introduce one's creative ideas or productions to others. In other words, fear of negative evaluation would not be correlated with creative achievements themselves, but with the tendency to expose these achievements to others' judgment. Second, interventions designed in order to enhance individuals' creativity could aim to reduce their apprehension concerning the judgements of others, which would lead them to feel more effective in their creativity, which would in turn lead them to engage in more creative and innovative behaviors (e.g., Hsu, Hou, \& Fan, 2011; Karwowski, Lebuda, Wisniewska, \& Gralewski, 2013; Puente-Díaz \& Karwowski, 2017).

There are, however, a few limitations that must be taken into account regarding the present contribution. First, our questionnaires included only self-reported measures of creativity. It has been shown that individuals possess awareness of their own level of creativity (e.g., self-rated creativity measures have been found to positively correlate with measures of creative potential and creative personality, see Furnham \& Bachtiar, 2008; Tyagi et al., 2017). However, one might regret the absence of creativity tasks in the present investigation. Our reasons for not including these was that no correlation between performance on such tasks and social risk-taking was observed by Tyagi et al. (2017). 
Future research should aim to further corroborate the associations between creativity, social risk-taking, and fear of negative evaluation using such tasks as measures of creative behaviors (e.g., the alternate uses task, see Acar \& Runco, 2019; Runco, Abdulla, Paek, Al-Jasim, \& Alsuwaidi, 2016). Such measures will provide insight on whether or not creative behaviors are related to the variables under consideration here. In particular, if these future studies confirm absence of association between creative behaviors and fear of negative evaluation, the factors explaining the inconsistency between the results related to self-beliefs, self-rated creativity, and behaviors will need to be investigated. We also observed a small-size negative association between SR and fear of negative evaluation of the individual $(r=-.14, p<.05)$, but not new ideas. Future research will need to establish whether this correlation is artifactual, as it is only significant at the threshold .05 (the p-value is one of the most predictive factors of successful replication, see Altmejd et al., 2019). Finally, this study did not aim to explore the causal link between our variables of interest. Future studies should explore such causal links through experimental or quasiexperimental methods (Dellas \& Gaier, 1970; Tyagi et al., 2017). They should also investigate a potential intervention of a third variable in the correlations studied here (Haig, 2003). For instance, personality traits have been found to correlate with both creativity (Furnham \& Bachtiar, 2008; Kaufman et al., 2016) and fear of negative evaluation (e.g., Hazel, Keaten, \& Kelly, 2014), and could thus be involved in the associations observed here.

In conclusion, in line with previous research, our results indicate that creative individuals seem to be social risk-takers. Creative individuals display stronger levels of social -taking, and individuals who are confident in their ability to generate creative productions seem to be less afraid of negative judgments from others. These findings thus reaffirm the importance of investigating the relationships between creativity and individual characteristics related to interpersonal and social interactions. Creative individuals display stronger levels of social risk-taking, and individuals who are confident in their ability to generate creative productions seem to be less afraid of negative judgments from others.

\section{REFERENCES}

Acar, S., \& Runco, M. A. (2019). Divergent thinking: New methods, recent research, and extended theory. Psychology of Aesthetics, Creativity, and the Arts, 13, 153-158. https://doi.org/10.1037/aca0000231

Amabile, T. M. (1996/2018). Creativity in Context: Update to the Social Psychology of Creativity. Boulder, CO: Westview Press.

Amabile, T. M., \& Pillemer, J. (2012). Perspectives on the social psychology of creativity. The Journal of Creative Behavior, 46, 3-15. https://doi.org/10.1002/jocb.001 
American Psychological Association (APA). (2017). Ethical principles of psychologists and code of conduct. http://www.apa.org/ethics/code/index.aspx

Batey, M., \& Furnham, A. (2008). The relationship between measures of creativity and schizotypy. Personality and Individual Differences, 45, 816-821. https:// doi.org/10.1016/j.paid.2008.08.014

Ben-Zur, H., \& Zeidner, M. (2009). Threat to life and risk-taking behaviors: A review of empirical findings and explanatory models. Personality and Social Psychology Review, 13, 109-128. https://doi.org/10.1177/1088868308330104

Blais, A. R., \& Weber, E. U. (2006). A domain-specific risk-taking (DOSPERT) scale for adult populations. Judgment and Decision making, 1, 33-47. https://doi.org/10.1037/ t13084-000

Bonetto, E., Pichot, N., Pavani, J.-B., \& Adam-Troïan, J. (2021). The paradox of creativity. New Ideas in Psychology, 60. https://doi.org/10.1016/j.newideapsych.2020.100820

Carleton, R. N., Collimore, K. C., \& Asmundson, G. J. (2007). Social anxiety and fear of negative evaluation: Construct validity of the BFNE-II. Journal of Anxiety Disorders, 21, 131-141. https://doi.org/10.1016/j.janxdis.2006.03.010

Carson, S. H., Peterson, J. B., \& Higgins, D. M. (2005). Reliability, validity, and factor structure of the creative achievement questionnaire. Creativity Research Journal, 17, 37-50. https://doi.org/10.1207/s15326934cri1701_4

Code de déontologie des psychologues (CNCDP) [Code of Ethics of Psychologists]. (1996, Amended February, 2012). http://www.cncdp.fr/index.php/code-dedeontologie/code-de-deontologie-2012

Csikszentmihalyi, M. (2015). The systems model of creativity: The collected works of Mihaly Csikszentmihalyi. New York, NY: Springer.

Dellas, M. \& Gaier, E. L. (1970). Identification of creativity: the individual. Psychol.Bull. 73, 55-73. doi: https://doi.org/10.1037/h0028446

Dollinger, S. J. (2003). Need for uniqueness, need for cognition, and creativity. The Journal of Creative Behavior, 37, 99-116. https://doi.org/10.1002/j.2162-6057.2003.tb00828.x

Funder, D. C., \& Ozer, D. J. (2019). Evaluating effect size in psychological research: Sense and nonsense. Advances in Methods and Practices in Psychological Science, 2, 156-168. https://doi.org/10.1177/2515245919847202

Furnham, A., \& Bachtiar, V. (2008). Personality and intelligence as predictors of creativity. Personality and Individual Differences, 45, 613-617. https://doi.org/10.1016/ j.paid.2008.06.023

Gough, H. G. (1979). A creative personality scale for the Adjective Check List. Journal of Personality and Social Psychology, 37, 1398-1405. https://doi.org/10.1037/0022-3514.37.8.1398 
Guilford, J. P. (1967). The Nature of Human Intelligence. New York, NY: McGraw-Hill.

Haig, B. D. (2003). What is a spurious correlation?. Understanding Statistics: Statistical Issues in Psychology, Education, and the Social Sciences, 2, 125-132. https:// doi.org/10.1207/S15328031US0202_03

Haslam, S. A., Adarves-Yorno, I., Postmes, T., \& Jans, L. (2013). The collective origins of valued originality: A social identity approach to creativity. Personality and Social Psychology Review, 17, 384-401. https://doi.org/10.1177/1088868313498001

Hazel, M., Keaten, J., \& Kelly, L. (2014). The relationship between personality temperament, communication reticence, and fear of negative evaluation. Communication Research Reports, 31, 339-347. https://doi.org/10.1080/08824096.2014.963219

Hsu, M. L., Hou, S. T., \& Fan, H. L. (2011). Creative self-efficacy and innovative behavior in a service setting: Optimism as a moderator. The Journal of Creative Behavior, 45, 258-272. https://doi.org/10.1002/j.2162-6057.2011.tb01430.x

Karwowski, M. (2012). Did curiosity kill the cat? Relationship between trait curiosity, creative self-efficacy and creative personal identity. Europe's Journal of Psychology, 8, 547-558. https://doi.org/10.5964/ejop.v8i4.513

Karwowski, M., \& Barbot, B. (2016). Creative self-beliefs: Their nature, development, and correlates. In J. C. Kaufman \& J. Baer (Eds.), Current perspectives in social and behavioral sciences. Creativity and reason in cognitive development (p. 302-326). Cambridge, UK: Cambridge University Press. https://doi.org/10.1017/ CBO9781139941969.016

Karwowski, M., Dul, J., Gralewski, J., Jauk, E., Jankowska, D. M., Gajda, A., ... \& Benedek, M. (2016). Is creativity without intelligence possible? A necessary condition analysis. Intelligence, 57, 105-117._https://doi.org/10.1016/j.intell.2016.04.006

Karwowski, M., \& Lebuda, I. (2016). The big five, the huge two, and creative self-beliefs: A meta-analysis. Psychology of Aesthetics, Creativity, and the Arts, 10(2), 214-232. https://doi.org/10.1037/aca0000035

Karwowski, M., Lebuda, I., Wisniewska, E., \& Gralewski, J. (2013). Big five personality traits as the predictors of creative self-efficacy and creative personal identity: Does gender matter?. The Journal of Creative Behavior, 47, 215-232. https://doi.org/10. 1002/jocb.32

Kaufman, S. B., Quilty, L. C., Grazioplene, R. G., Hirsh, J. B., Gray, J. R., Peterson, J. B., \& DeYoung, C. G. (2016). Openness to experience and intellect differentially predict creative achievement in the arts and sciences. Journal of Personality, 84, 248-258. https://doi.org/10.1111/jopy.12156 
Kim, K. H. (2005). Can only intelligent people be creative? A meta-analysis. Journal of Secondary Gifted Education, 16, 57-66. https://doi.org/10.4219/jsge-2005-473

Leary, M. R. (1983). A brief version of the Fear of Negative Evaluation Scale. Personality and Social Psychology Bulletin, 9, 371-375. https://doi.org/10.1177/0146167283093007 Lucas, B. J., \& Nordgren, L. F. (2020). The creative cliff illusion. Proceedings of the National Academy of Sciences, 117, 19830-19836. https://doi.org/10.1073/pnas. 2005620117

Mumford, M. D. (2012). Handbook of Organizational Creativity. London, UK: Academic Press. Puente-Díaz, R., \& Karwowski, M. (2017). Creative self-beliefs and their implications for creativity and innovation. In A. Brem, R. Puente-Díaz, \& M. Agogué (Eds.), The Role of Creativity in the Management of Innovation: State of the Art and Future Research Outlook (pp.149-158). London, UK: World Scientific.

Runco, M. A., Abdulla, A. M., Paek, S. H., Al-Jasim, F. A., \& Alsuwaidi, H. N. (2016). Which test of divergent thinking is best?. Creativity. Theories-ResearchApplications, 3, 4-18. https://doi.org/10.1515/ctra-2016-0001

Runco, M. A., \& Jaeger, G. J. (2012). The standard definition of creativity. Creativity research journal, 24, 92-96. https://doi.org/10.1080/10400419.2012.650092

Runco, M. A., Plucker, J. A., \& Lim, W. (2001). Development and psychometric integrity of a measure of ideational behavior. Creativity Research Journal, 13, 393-400. https://doi.org/10.1207/S15326934CRJ1334_16

Sternberg, R. J. (1999). Handbook of Creativity. Cambridge, UK: Cambridge University Press. Tyagi, V., Hanoch, Y., Hall, S. D., Runco, M., \& Denham, S. L. (2017). The risky side of creativity: Domain specific risk taking in creative individuals. Frontiers in psychology, 8, 145. https://doi.org/10.3389/fpsyg.2017.00145

Watts, L. L., Steele, L. M., \& Song, H. (2017). Re-examining the relationship between need for cognition and creativity: Predicting creative problem solving across multiple domains. Creativity Research Journal, 29, 21-28. https://doi.org/10.1080/10400 419.2017.1263505

Corresponding author at: Eric Bonetto, Aix Marseille Univ, LPS, Aix-en-Provence, France, Aix Marseille Univ, PSYCLE (Center for Research on the Psychology of Cognition, Language and Emotion), Aix-en-Provence, France. InCIAM (Aix-Marseille Institute of Creativity and Innovation), Aix-en-Provence, France

E-mail: bonetto.ericbw@gmail.com

(C) Copyright by Faculty of Education, University of Bialystok, 